\title{
Efeitos da massagem terapêutica em recém-nascidos prematuros na unidade de terapia intensiva neonatal: uma revisão sistemática
}

\author{
Effects of therapeutic massage on premature newborn in the \\ neonatal intensive therapy unit: a systematic review
}

\author{
André Luiz Lisboa Cordeiro', Cindy Luara Morais de Andrade ${ }^{2}$, Filipe Souza França ${ }^{3}$, \\ Matheus Carvalho e Carvalho4, Larissa Maria Menezes Pinto ${ }^{5}$ \\ ${ }^{1}$ Autor correspondente. Faculdade Nobre, Escola Bahiana de Medicina e Saúde Pública, Feira de Santana, Salvador, Bahia, Brasil. \\ ORCID: 0000-0002-8126-8644. andrelisboacordeiro@gmail.com \\ ${ }^{2}$ Faculdade Nobre, Feira de Santana, Bahia, Brasil. ORCID: 0000-0002-1749-5164. cindyandradee@gmail.com \\ ${ }^{3}$ Faculdade Nobre, Feira de Santana, Bahia, Brasil. ORCID: 0000-0001-5426-8182. filipesoouza25@gmail.com \\ ${ }^{4}$ Faculdade Nobre, Feira de Santana, Bahia, Brasil. ORCID: 0000-0002-3272-1350. mattheus.c@outlook.com \\ ${ }^{5}$ Faculdade Nobre, Feira de Santana, Bahia, Brasil. ORCID: 0000-0002-3141-704X. larihmenezes@hotmail.com
}

RESUMO | INTRODUÇÃO: A Unidade de Terapia Intensiva Neonatal (UTIN) é o local para recém-nascidos de risco que necessitam de cuidados contínuos. A massagem terapêutica é um toque sistemático das mãos humanas que contribui para o ganho de peso, diminui as respostas à dor e diminui a permanência no hospital. OBJETIVO: Revisar os efeitos sistêmicos da massagem terapêutica em prematuros $\mathrm{Na}$ Unidade de Terapia Intensiva Neonatal. METOdologIA: Esta é uma revisão sistemática. Foi realizada uma busca nas bases de dados Pubmed, Scielo e Lilacs, sem restrições relacionadas ao ano de publicação. Apenas ensaios clínicos randomizados foram incluídos neste estudo, com uma amostra de indivíduos de ambos os sexos, com menos de 28 dias de idade e abordando massoterapia relacionada a respostas sistêmicas em prematuros na unidade de terapia intensiva neonatal. Foram excluídos os estudos relacionados ao exercício físico, uso de medicamentos, estimulação oral e efeitos gastrointestinais relacionados à massagem. Foram utilizados os seguintes descritores: "massagem" AND "recém-nascido prematuro" OR "prematuro" OR "prematuro" AND "unidades de terapia intensiva neonatal". RESULTADOS: Dos seis artigos incluídos nesta revisão, cinco apresentaram resultados satisfatórios sobre os efeitos sistêmicos da massagem terapêutica em recém-nascidos prematuros, como ganho de peso, diminuição da permanência na UTIN, melhora da função do sistema nervoso parassimpático e aumento da citotoxicidade celular de células assassinas naturais. Apenas um estudo relata que a massagem terapêutica não induz o sono. CONCLUSÃO: A massagem terapêutica produz efeitos benéficos sistêmicos em prematuros internados na Unidade de Terapia Intensiva Neonatal.
ABSTRACT | INTRODUCTION: The Neonatal Intensive Care Unit (NICU) is the place for at-risk newborns who need ongoing care. Massage therapy is a systematic touch by human hands that contributes to weight gain, decreases pain responses and decreases hospital stay. OBJECTIVE: To review the systemic effects of massage therapy on premature infants in the Neonatal Intensive Care Unit. METHODOLOGY: This is a systematic review. A search was performed in the PubMed, SCIELO and LILACS database, with no restrictions related to the year of publication. Only randomized clinical trials were included in this study, with a sample of individuals of both sexes, under 28 days of age and addressing massage therapy related to systemic responses in premature infants in the neonatal intensive care unit. Studies that were related to physical exercise, medication use, oral stimulation and gastrointestinal effects related to massage were excluded. The following descriptors were used: "massage" AND "premature newborn" OR "premature" OR "preterm" AND "neonatal intensive care units". RESULTS: Of the six articles included in this literature review, five showed satisfactory results on the systemic effects of massage therapy on premature newborns, such as weight gain, decreased length of stay in the NICU, improvement in parasympathetic nervous system function and increased cell cytotoxicity of natural killer cells. Only one study reports that massage therapy does not induce sleep. CONCLUSION: Massage therapy produces systemic beneficial effects in preterm infants admitted to the Neonatal Intensive Care Unit.

KEYWORDS: Infant. Premature. Massage. Intensive Care units neonatal.

PALAVRAS-CHAVE: Infância. Prematuro. Massagem. Unidades de

terapia intensiva neonatal. 


\section{Introdução}

A prematuridade é considerada um problema de saúde pública devido ao alto índice de morbimortalidade infantil' ${ }^{1}$. Bebês prematuros são aqueles nascidos antes das 37 semanas de gestação, $11,8 \%$ dos nascidos vivos no Brasil. Segundo a Organização Mundial da Saúde (OMS), a principal causa de morte em crianças menores de 5 anos foi o parto prematuro².

Devido à imaturidade dos órgãos e sistemas, os recém-nascidos terão dificuldade em se adaptar à vida extrauterina. Mas, dependendo do grau de prematuridade e das complicações associadas, nem todos os bebês precisarão de cuidados nas Unidades de Terapia Intensiva Neonatal (UTIN) ${ }^{3}$. A UTIN é o local para recém-nascidos de risco que precisam de cuidados contínuos. É um ambiente com alto grau de tecnologia, contribuindo para a sobrevivência de recém-nascidos ${ }^{4}$.

No entanto, a UTIN torna-se um ambiente estressante para os recém-nascidos porque, além das consequências fisiológicas da prematuridade, estão expostos à luz, ruído e cuidados médicos que levam a alterações nas funções sensoriais, motoras e cognitivas ${ }^{5}$.

O papel do fisioterapeuta na UTIN varia de acordo com o paciente, mas é caracterizado pelo manuseio motor, manobras pulmonares e posições terapêuticas ${ }^{6,7}$. A fisioterapia motora engloba vários estímulos táteis, vestibulares, proprioceptivos, visuais e auditivos, facilitando o desenvolvimento neuropsicomotor do recém-nascido ${ }^{6}$.

Nesse contexto, a massagem terapêutica é definida como um toque sistemático pelas mãos humanas, que gera uma estimulação tátil do recém-nascido prematuro, o que contribui para o ganho de peso, diminui as respostas à dor, melhora a digestão, diminui o gasto energético e diminui o tempo de internação?

Existem vários protocolos em relação ao tipo de massagem e muitas vezes são combinados com outras formas de estimulação, algumas envolvendo pressão moderada com estímulos táteis e também associadas a estímulos cinestésicos onde são realizados movimentos passivos de flexão e extensão dos braços e pernas ${ }^{8}$.
Assim, este estudo tem como objetivo revisar os efeitos sistêmicos da massagem terapêutica em prematuros na Unidade de Terapia Intensiva Neonatal (UTIN).

\section{Materiais e métodos}

\section{Estratégia de identificação da busca de estudo}

Trata-se de uma revisão sistemática realizada na pesquisa no banco de dados MEDLINE (PubMed), na biblioteca virtual de saúde (LILACS) e na Scientific Electronic Library Online (Scielo). Foram selecionados artigos em português, inglês e espanhol, sem restrições relacionadas ao ano de publicação. A busca de artigos foi realizada de fevereiro a maio de 2019.

Os descritores utilizados foram: "massoterapia" and "recém-nascido prematuro" or "prematuro" "pré-termo" and "unidades de terapia intensiva neonatal".

\section{Critérios de elegibilidade}

Este estudo incluiu apenas ensaios clínicos randomizados publicados com uma amostra de indivíduos de ambos os sexos, com menos de 28 dias de idade e abordando massagem terapêutica relacionada a respostas sistêmicas em prematuros na unidade de terapia intensiva neonatal.

Foram excluídos os estudos relacionados ao exercício físico, uso de medicamentos, estimulação oral e efeitos gastrointestinais relacionados à massagem.

\section{Avaliação da qualidade metodológica}

A qualidade metodológica foi avaliada de acordo com o Manual Cochrane de Revisões Sistemáticas de Intervenções, versão 5.1.0. Os estudos foram avaliados de acordo com os sete domínios: tipos de randomização; sigilo de alocação; cegueira; análise de intenção de tratar; parada antecipada para benefício; descrição seletiva do resultado; escala validada. O risco de viés foi classificado como "baixo risco de viés" quando era muito claro nesses domínios, "alto risco de viés" quando não mencionado e "risco incerto de viés" se a informação existisse, mas sem clareza. 


\section{Extração dos dados}

Para extração dos artigos selecionados, verificamos títulos e resumos. Posteriormente, os artigos foram comparados com os critérios de inclusão pré-estabelecidos, a fim de obter resultados para a revisão sistemática.

Dois revisores avaliaram independentemente a qualidade metodológica dos estudos, o resultado foi comparado e discutido até que a concordância fosse alcançada. Se houvesse discordância entre os avaliadores os estudos seriam enviados para um terceiro avaliador.

\section{Resultados}

A partir dos critérios de busca, inicialmente 25 estudos foram detectados. Após a leitura dos artigos selecionados, 19 foram excluídos devido à presença de critérios (2 por haver massagem terapêutica associada à estimulação oral, 12 associados a medicamentos e 5 envolvendo exercício físico), totalizando seis estudos para análise final (Figura 1).

Figura 1. Fluxograma para obtenção dos resultados

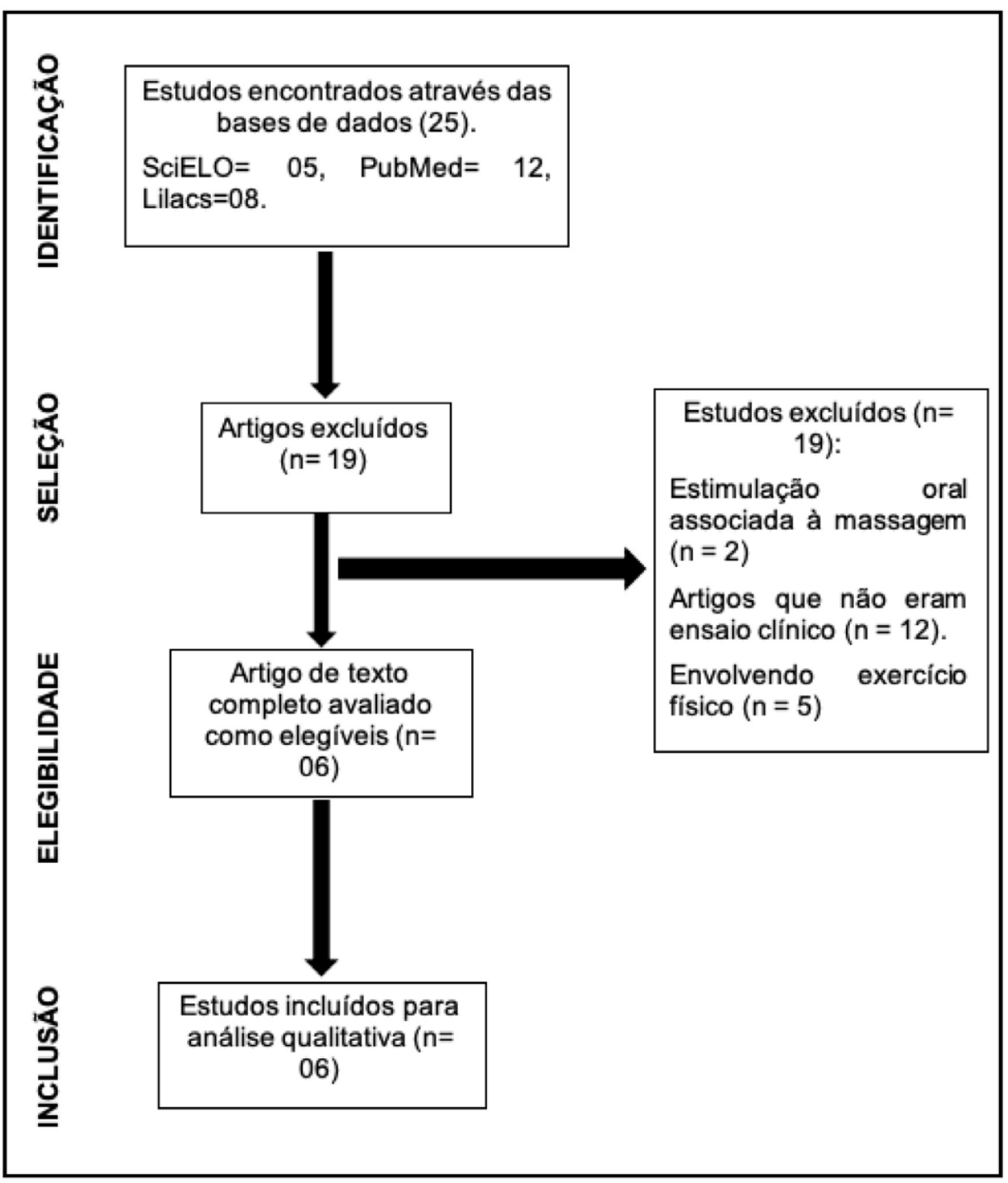


A qualidade metodológica desses seis artigos foi analisada usando a ferramenta Cochrane Collaboration (Quadro 1). Os artigos foram avaliados com os seguintes critérios: alto risco de viés, baixo risco de viés e risco incerto de viés.

Os seis estudos incluídos nesta revisão sistemática discutem os efeitos sistêmicos da massagem terapêutica em prematuros na UTI neonatal.

Quadro 1. Análise metodológica da qualidade dos estudos: risco de viés de cada estudo com base na ferramenta Cochrane Collaboration

\begin{tabular}{|c|c|c|c|c|c|c|}
\hline & $\begin{array}{l}\text { Abdallah et } \\
\text { al. }^{7}\end{array}$ & Ang et al. ${ }^{9}$ & $\begin{array}{l}\text { Massaro et } \\
\text { al. }^{10}\end{array}$ & Smith et al. ${ }^{11}$ & Taheri et al. $^{12}$ & Yates et al. ${ }^{13}$ \\
\hline Tipo de Randomização & $\begin{array}{l}\text { Baixo risco de } \\
\text { viés }\end{array}$ & $\begin{array}{l}\text { Baixo risco de } \\
\text { viés }\end{array}$ & $\begin{array}{l}\text { Baixo risco de } \\
\text { viés }\end{array}$ & $\begin{array}{l}\text { Baixo risco de } \\
\text { viés }\end{array}$ & $\begin{array}{l}\text { Baixo risco de } \\
\text { viés }\end{array}$ & $\begin{array}{l}\text { Baixo risco de } \\
\text { viés }\end{array}$ \\
\hline Sigilo de alocações & $\begin{array}{l}\text { Incerto risco de } \\
\text { viés }\end{array}$ & $\begin{array}{l}\text { Incerto risco } \\
\text { de viés }\end{array}$ & $\begin{array}{l}\text { Alto risco de } \\
\text { viés }\end{array}$ & $\begin{array}{l}\text { Incerto risco } \\
\text { de viés }\end{array}$ & $\begin{array}{ll}\text { Incerto risco } \\
\text { de viés }\end{array}$ & $\begin{array}{l}\text { Incerto risco de } \\
\text { viés }\end{array}$ \\
\hline Cegamento & $\begin{array}{l}\text { Baixo risco de } \\
\text { viés }\end{array}$ & $\begin{array}{l}\text { Alto risco de } \\
\text { viés }\end{array}$ & $\begin{array}{l}\text { Baixo risco de } \\
\text { viés }\end{array}$ & $\begin{array}{l}\text { Baixo risco de } \\
\text { viés }\end{array}$ & $\begin{array}{l}\text { Alto risco de } \\
\text { viés }\end{array}$ & $\begin{array}{l}\text { Incerto risco de } \\
\text { viés }\end{array}$ \\
\hline $\begin{array}{c}\text { Análise de intenção de } \\
\text { tratar }\end{array}$ & $\begin{array}{l}\text { Baixo risco de } \\
\text { viés }\end{array}$ & $\begin{array}{l}\text { Baixo risco de } \\
\text { viés }\end{array}$ & $\begin{array}{l}\text { Baixo risco de } \\
\text { viés }\end{array}$ & $\begin{array}{l}\text { Baixo risco de } \\
\text { viés }\end{array}$ & $\begin{array}{l}\text { Baixo risco de } \\
\text { viés }\end{array}$ & $\begin{array}{l}\text { Baixo risco de } \\
\text { viés }\end{array}$ \\
\hline $\begin{array}{c}\text { Parada antecipada para } \\
\text { benefício }\end{array}$ & $\begin{array}{l}\text { Baixo risco de } \\
\text { viés }\end{array}$ & $\begin{array}{l}\text { Baixo risco de } \\
\text { viés }\end{array}$ & $\begin{array}{l}\text { Baixo risco de } \\
\text { viés }\end{array}$ & $\begin{array}{l}\text { Baixo risco de } \\
\text { viés }\end{array}$ & $\begin{array}{l}\text { Baixo risco de } \\
\text { viés }\end{array}$ & $\begin{array}{l}\text { Baixo risco de } \\
\text { viés }\end{array}$ \\
\hline $\begin{array}{c}\text { Descrição seletiva do } \\
\text { resultado }\end{array}$ & $\begin{array}{l}\text { Incerto risco de } \\
\text { viés }\end{array}$ & $\begin{array}{l}\text { Baixo risco de } \\
\text { viés }\end{array}$ & $\begin{array}{l}\text { Incerto risco de } \\
\text { viés }\end{array}$ & $\begin{array}{l}\text { Alto risco de } \\
\text { viés }\end{array}$ & $\begin{array}{l}\text { Alto risco de } \\
\text { viés }\end{array}$ & $\begin{array}{l}\text { Baixo risco de } \\
\text { viés }\end{array}$ \\
\hline Escala validada & $\begin{array}{l}\text { Incerto risco de } \\
\text { viés }\end{array}$ & $\begin{array}{l}\text { Incerto risco } \\
\text { de viés }\end{array}$ & $\begin{array}{l}\text { Alto risco de } \\
\text { viés }\end{array}$ & $\begin{array}{l}\text { Alto risco de } \\
\text { viés }\end{array}$ & $\begin{array}{ll}\text { Incerto risco } \\
\text { de viés }\end{array}$ & $\begin{array}{l}\text { Incerto risco de } \\
\text { viés }\end{array}$ \\
\hline
\end{tabular}

Dos seis artigos incluídos nesta revisão de literatura, cinco apresentaram resultados satisfatórios sobre os efeitos sistêmicos da massagem terapêutica em recém-nascidos prematuros, como ganho de peso, diminuição da permanência na UTIN, melhora da função e desenvolvimento do sistema nervoso parassimpático e aumento da célula citotoxicidade de células assassinas naturais. Apenas um estudo relata que a massagem terapêutica não induz o sono imediatamente após a massagem. A quadro 2 mostra os principais ensaios clínicos sobre o assunto e seus resultados. 
Quadro 2. Dados gerais sobre os artigos selecionados para obtenção dos resultados

\begin{tabular}{|c|c|c|c|c|}
\hline Autor & Objetivo & Amostra & Intervenção & Conclusão \\
\hline Massaro et al. ${ }^{10}$ & $\begin{array}{l}\text { Avaliar os efeitos da } \\
\text { massagem com ou sem } \\
\text { estimulação cinestésica no } \\
\text { ganho de peso prematuro. }\end{array}$ & $\begin{array}{l}\text { GC: } 20 . \\
\text { GI1: } 20 . \\
\text { GI2: } 20\end{array}$ & $\begin{array}{l}\text { GC: Bebês gerenciados por } \\
\text { meio de atendimento padrão } \\
\text { no berçário. } \\
\text { GC1: Massagem terapêutica } \\
\text { infantil com estimulação tátil } \\
\text { cinestésica. } \\
\text { GI2: Massagem sem } \\
\text { estimulação tátil cinestésica. }\end{array}$ & $\begin{array}{c}\text { A massagem com estimulação } \\
\text { tátil cinestésica está associada } \\
\text { a uma melhora no ganho de } \\
\text { peso diário em recém- } \\
\text { nascidos. }\end{array}$ \\
\hline Abdallah et al. ${ }^{7}$ & $\begin{array}{l}\text { Avaliar os benefícios a curto } \\
\text { e longo prazo da massagem } \\
\text { em prematuros estáveis. }\end{array}$ & $\begin{array}{l}\text { Gl: } 32 . \\
\text { GC: } 34 .\end{array}$ & $\begin{array}{c}\text { GI: Massagem terapêutica } \\
\text { infantil com duração de } 10 \\
\text { minutos. } \\
\text { GC: Tratamento idêntico em } \\
\text { todas as áreas de } \\
\text { atendimento da UTIN, exceto } \\
\text { na intervenção de } \\
\text { massagem. }\end{array}$ & $\begin{array}{l}\text { Os resultados deste estudo } \\
\text { indicam que a massagem é } \\
\text { benéfica para bebês } \\
\text { prematuros estáveis. }\end{array}$ \\
\hline Yates et al. ${ }^{13}$ & $\begin{array}{l}\text { O objetivo deste estudo foi } \\
\text { determinar se a massagem } \\
\text { terapêutica pode ser usada } \\
\text { como um complemento } \\
\text { para induzir o sono em } \\
\text { bebês prematuros. }\end{array}$ & $\begin{array}{l}\text { MT1: } 10 . \\
\text { MT2: } 13 .\end{array}$ & $\begin{array}{l}\text { MT1: Recebeu massagem } \\
\text { terapêutica por } 10 \text { minutos } \\
\text { pela manhã no dia } 1 \text { do } \\
\text { estudo e não recebeu } \\
\text { massagem no dia } 2 \text { do } \\
\text { estudo. } \\
\text { MT2: O segundo grupo não } \\
\text { recebeu massagem no dia } 1 \\
\text { e fez massagem terapêutica } \\
\text { pela manhã no dia } 2 .\end{array}$ & $\begin{array}{c}\text { A massagem terapêutica não } \\
\text { induz o sono imediatamente } \\
\text { após a massagem. }\end{array}$ \\
\hline Taheri et al. ${ }^{12}$ & $\begin{array}{l}\text { Avaliar a eficácia do ciclo de } \\
\text { cinco dias do óleo de } \\
\text { girassol em } \\
\text { massagem com pressão } \\
\text { moderada sobre ganho de } \\
\text { peso e tempo de internação } \\
\text { na UTIN em prematuros. }\end{array}$ & $\begin{array}{c}\text { Gl: } 22 . \\
\text { GC: } 22 .\end{array}$ & $\begin{array}{l}\text { Gl: Massagem com óleo de } \\
\text { girassol com pressão } \\
\text { moderada. Cada sessão de } \\
15 \text { minutos consiste em três } \\
\text { etapas consecutivas de cinco } \\
\text { minutos. } \\
\text { GC: recebeu apenas } \\
\text { atendimento de rotina na } \\
\text { UTIN. }\end{array}$ & $\begin{array}{l}\text { Mesmo um curto período de } \\
\text { massagem corporal com óleo } \\
\text { de girassol aumenta o ganho } \\
\text { de peso em bebês prematuros } \\
\text { e diminui o tempo de } \\
\text { permanência na UTIN. }\end{array}$ \\
\hline Ang et al. ${ }^{9}$ & $\begin{array}{l}\text { Investigar os efeitos da } \\
\text { massagem terapêutica no } \\
\text { sistema imunológico de } \\
\text { recém-nascidos } \\
\text { prematuros. }\end{array}$ & $\begin{array}{l}\text { GC: } 62 . \\
\text { GI: } 58 .\end{array}$ & $\begin{array}{l}\text { Gl: Massagem Terapêutica. } \\
\text { Cada sessão durou } 15 \\
\text { minutos e consistiu em três } \\
\text { fases de } 5 \text { minutos. } \\
\text { GC: A enfermeira da pesquisa } \\
\text { permaneceu atrás das duas } \\
\text { telas grandes e passou o } \\
\text { mesmo tempo sem contato } \\
\text { físico com as crianças. }\end{array}$ & $\begin{array}{l}\text { Associação positiva entre } \\
\text { citotoxicidade da massagem } \\
\text { terapêutica e células natural } \\
\text { killer (NK), bem como } \\
\text { massagem terapêutica e ganho } \\
\text { de peso em prematuros. }\end{array}$ \\
\hline Smith et al. ${ }^{11}$ & $\begin{array}{c}\text { Teste a hipótese de que a } \\
\text { massagem melhoraria a } \\
\text { função do sistema nervoso } \\
\text { autônomo (SNA) através da } \\
\text { variabilidade da frequência } \\
\text { cardíaca (VFC) em bebês } \\
\text { prematuros. }\end{array}$ & $\begin{array}{l}\text { GI: } 17 . \\
\text { GC: } 20 .\end{array}$ & $\begin{array}{l}\text { GI: Protocolo de Massagem } \\
\text { Infantil Modificado. Foi } \\
\text { realizado por } 20 \text { minutos, } \\
\text { duas vezes ao dia. } \\
\text { GC: Os bebês de controle } \\
\text { estavam em decúbito dorsal, } \\
\text { mas não receberam } \\
\text { massagem. }\end{array}$ & $\begin{array}{l}\text { Houve um efeito positivo da } \\
\text { massagem na função e } \\
\text { desenvolvimento } \\
\text { parassimpático do SNA. }\end{array}$ \\
\hline
\end{tabular}

GC - Grupo Controle; IG - Grupo de Intervenção; MT - massagem terapêutica; VFC - variabilidade da frequência cardíaca; SNA - Sistema Nervoso Autonômico; NK - Células Assassinas Naturais; UTIN - Unidades de Terapia Intensiva Neonatal. 


\section{Discussão}

Esta revisão sistemática propôs compreender os efeitos sistêmicos da massagem terapêutica na UTIN. Embora a UTIN seja um ambiente estressante devido ao seu ruído excessivo, iluminação forte, que gera algumas mudanças comportamentais nos recém-nascidos, descobrimos que as massagens terapêuticas estão relacionadas ao ganho de peso, redução do tempo de internação e melhora da função. SNA parassimpático de recém-nascidos.

Estudos apontam vários benefícios, no entanto, existem muitas variações em termos de técnica de massagem, algumas com pressão moderada, outras com estimulação tátil cinestésica, características dos recém-nascidos, aplicação de óleos, diferentes técnicas e duração do estudo.

Abdallah et al. ${ }^{7}$ encontraram no estudo que a massagem terapêutica das mães antes de uma punção no calcanhar teve um efeito positivo nos escores de dor pós-massagem nos grupos de intervenção e também teve um escore menor quando comparado ao grupo controle na alta. E, de acordo com o estudo de Taheri et al. ${ }^{12}$, a massagem corporal associada à estimulação tátil cinestésica e à aplicação de óleo de girassol ao longo de cinco dias não apenas aumentou o peso e o ganho dos bebês, mas também diminuiu o tempo de permanência destes em UTIN. Encurtar esse período de hospitalização dos recém-nascidos, além de rentável, reduz a riqueza de uma possível complicação durante o período de hospitalização.

No estudo de Massaro et al. ${ }^{10}$, a massagem terapêutica associada à estimulação tátil cinestésica também gerou aumento de ganho de peso em recém-nascidos prematuros. Esse resultado provavelmente está relacionado à melhora da função metabólica basal, uma vez que os bebês que receberam massagem ou massagem com estimulação cinestésica não consomem ou retêm mais calorias que os grupos controle, a ingestão calórica nos estudos tem um padrão semelhante para todos. Ou seja, essa eficiência metabólica gera aquisição de ganho de massa corporal.

O estudo de Yates et al. ${ }^{13}$ teve como objetivo determinar se a massagem promoveria relaxamento para induzir o sono imediatamente após a massagem, constatando que a massagem estimula a atividade elétrica cerebral, promovendo a maturação do cérebro.
Embora a massagem terapêutica neste estudo não induza o sono imediatamente após a massagem, os benefícios estão associados à sua aplicação ao aumento da citotoxicidade das células assassinas naturais. Essas células são linfócitos que possuem atividade citolítica, ou seja, têm a função de destruir células tumorais ou células infectadas por vírus. Ainda assim, o estudo de Ang et al. ${ }^{9}$ mostrou que a massagem terapêutica tem uma associação positiva com a citotoxicidade natural das células assassinas, e foram maiores no grupo intervenção quando comparado ao grupo controle.

O estudo de Smith et al. ${ }^{11}$ teve como objetivo testar a hipótese de que a massagem melhoraria a função do sistema nervoso autônomo (SNA), medida pela variabilidade da frequência cardíaca (VFC), mostrando que em bebês prematuros essa resposta será exagerada devido à imaturidade do ramo parassimpático. No entanto, o estudo de Smith et al. ${ }^{11}$ mostra que a massagem terapêutica desenvolveu a função parassimpática de recém-nascidos prematuros no grupo intervenção.

Portanto, é claro que a massagem bem administrada pode melhorar o desenvolvimento de SNA de prematuros mais suscetíveis à resposta ao estresse.

As limitações deste estudo são, principalmente quanto à apresentação das variáveis e dos seus parâmetros. Além disto, o tamanho amostral dos estudos variou de forma considerável, o que também contribui como limitação.

\section{Conclusão}

Os resultados mostram que a massagem terapêutica é benéfica para recém-nascidos prematuros, mostrando efeitos positivos em seu crescimento, principalmente no ganho de peso. Também está associado à diminuição do tempo de permanência na UTIN, melhora na função e desenvolvimento do SNA parassimpático e aumento da citotoxicidade celular, mostrando-se uma técnica promissora em ambientes com poucos recursos. 


\section{Contribuições do autor}

Cordeiro ALL participou da concepção e design da pesquisa; coleta, análise e interpretação dos dados, redação e revisão crítica do manuscrito. Andrade C, França F, Carvalho M participou da concepção e desenho da pesquisa, coleta de dados e redação do manuscrito. L. Menezes participou da redação e revisão crítica do manuscrito.

\section{Conflito de interesses}

Não foram divulgados interesses financeiros, legais ou políticos em concorrência com terceiros (governo, comércio, fundação privada, etc.) para qualquer aspecto do trabalho enviado (incluindo, entre outros, subsídios, conselho de monitoramento de dados, desenho de estudo, preparação de manuscrito, estatística, análise, etc.).

\section{Referências}

1. Klossowski DG, Godoi VC, Xavier CR, Fujinaga CRX. Assistência integral ao recém-nascido prematuro: implicações das práticas e da política pública. Rev. CEFAC. 2016;18(1): 137-150. doi: 10.1590/1982-021620161814515

2. Martins SW, Enumo SRF, Paula KMP. Manejo da dor neonatal: influência de fatores psicológicos e organizacionais. Estud Psicol. 2016;33(4):633-44. doi: 10.1590/1982-02752016000400007

3. Mota LA, Sá FE, Frota MA. Estudo Comparativo do Desenvolvimento Sensório-Motor de Recém-Nascidos Prematuros da Unidade de Terapia Intensiva Neonotal e do Método Canguru. Rev Brasileira em Promoção da Saúde. 2005;18(4):191-198.

4. Silva LJ, Silva LR, Christoffel MM. Tecnologia e humanização na Unidade de Terapia Intensiva Neonatal: reflexões no contexto do processo saúde-doença. Rev. Esc. Enferm. USP. 2009;43(3):684-9. doi: 10.1590/S0080-62342009000300026

5. Pineda RG, Neil J, Dierker D, Smyser CD, Wallendorf $\mathrm{M}$, Kikodoro $\mathrm{H}$ et al. Alterations in brain structure and neurodevelopmental outcome in preterm infants hospitalized in different neonatal intensive care unit environments. Journal of Pediatrics. 2014;164(1):52-60. doi: 10.1016/j.jpeds.2013.08.047

6. Theis RCSR, Gerzson LR, Almeida CS. A atuação do profissional fisioterapeuta em Unidades de Terapia Intensiva Neonatal. Rev. depart. ed física e saúde. 2016; 17(2);168-176. doi: $10.17058 /$ cinergis.v17i2.7703

7. Abdallah B, Badr LK, Hawwari M. The efficacy of massage on short and long term outcomes in preterm infants. Infat Behavior and Development. 2013;36(4);662-9. doi: 10.1016/j. infbeh.2013.06.009

8. Field T, Diego M, Hernandez-Reif M. Preterm infant massage therapy research: A review. Infant Behavior and Development. 2010;33(2);115-124. doi: 10.1016/j.infbeh.2009.12.004

9. Ang JY, Lua JL, Mathur A, Thomas R, Asmar BI, Savasan S et al. A randomized placebo-controlled trial of massage therapy on the immune system of preterm infants. Pediatrics. 2012;130(6);15491558. doi: $10.1542 /$ peds.2012-0196

10. Massaro AN, Hammad TA, Jazzo B, Aly H. Massage with kinesthetic stimulation improves weight gain in preterm infants. Journal of perinatology. 2009;29(5);352-7. doi: 10.1038/ j.j. 2008.230

11. Smith SL, Lux R, Haley S, Slater H, Beechy J, Moyer-Mileur LJ. The effect of massage on heart rate variability in preterm infants. Journal of Perinatology. 2013; 33(1);59-64. doi: 10.1038/jp.2012.47

12. Taheri PA, Goudarzi Z, Shariat M, Nariman S, Matin EN. The effect of a short course of moderate pressure sunflower oil massage on the weight gain velocity and length of NICU stay in preterm infants. Infant Behav Dev. 2018;5(1);22-27. doi: 10.1016/j. infbeh.2017.11.002

13. Yates CC, Mitchell AJ, Booth MY, Williams DK, Lowe LM, With Hall R. The effects of massage therapy to induce sleep in infants born preterm. Pediatric Phys Ther. 2014;26(4);405-410. doi: 10.1097/PEP.0000000000000081 